\title{
Mastalgias Cíclicas: Tratamento Não-Medicamentoso (Orientação Verbal)
}

\author{
Cyclical Mastalgias: A Nonpharmacological Treatment (Reassurance) \\ Flávio Nunes Sivini ${ }^{1}$, Aurélio Molina ${ }^{1}$, \\ Cícero Ferreira Fernandes Costa ${ }^{1}$, Flávio Murilo Pinto Sivini ${ }^{2}$
}

\begin{abstract}
RESUMO
Objetivos: avaliar o tratamento não-medicamentoso (orientação verbal) como primeira opção terapêutica para mulheres com mastalgia cíclica e observar se o tempo de existência da sintomatologia dolorosa altera os resultados.

Métodos: conduzimos um estudo do tipo experimental não-controlado com uma amostra de 128 mulheres com história clara de mastalgia cíclica, tratadas com orientação verbal. Uma escala analógica visual da dor foi usada antes e após o tratamento, a fim de avaliar a severidade, e classificamos as mastalgias em graus I (leve), II (moderado) e III (severo), de acordo com a intensidade da dor. Usamos também o "Cardiff Breast Score" (CBS) modificado para avaliar a resposta clinica ao tratamento. A análise dos dados foi feita com o teste do $\chi^{2}$ (Epi-Info 6.04).

Resultados: verificou-se um indice de sucesso de 59,4\% com a orientação verbal, mas não houve resposta com diferença estatisticamente significante entre os grupos $(p=0,16)$ com diferentes graus de mastalgia. A resposta menos satisfatória ao tratamento não-medicamentoso nas pacientes que apresentavam um periodo de tempo mais longo de sintomatologia foi apenas aparente, pois não houve diferença estatisticamente significante $(p=0.14)$.

Conclusão: a orientação verbal deve ser tentada sempre como a primeira escolha terapêutica para mulheres com mastalgia cíclica, independentemente do grau de intensidade da dor. $O$ curso prolongado da dor não interferiu nos resultados.
\end{abstract}

PALAVRAS CHAVE: Mama: doenças benignas. Mastalgia, orientação verbal

\section{Introdução}

Mastalgia é o termo empregado para definir a dor localizada na mama e deve ser considerada como um sintoma mais do que uma doença ${ }^{1}$. É, sem dúvida, a queixa mais freqüente das pacientes em relação às suas glândulas mamárias, e também a causa mais comum de consultas aos ambulatórios de patologia mamária ${ }^{2}$.

Pode ser classificada em cíclica, não-cíclica e não-mamária. A mastalgia cíclica por definição, é aquela que ocorre no menacme. A dor tem uma relação clara com o ciclo menstrual, geralmente ocorrendo na fase pré-menstrual e acabando com a menstruação ${ }^{3}$.

${ }^{1}$ Departamento Materno-Infantil da Faculdade de Ciências Médicas da Universidade de Pernambuco.

${ }^{2}$ Instituto de Ciências Biológicas da Faculdade de Ciências

Médicas da Universidade de Pernambuco.

Correspondência:

Flávio Nunes Sivini

Av. Boa Viagem, 5600 ap. 302

51030-000 - Recife - Pernambuco
A mastalgia, para a maioria das pacientes, é um problema autolimitado e suave, mas para outras, em menor proporção, a dor afeta seu trabalho, sua vida sexual e familiar ${ }^{4}$. Depressão, perda de auto-estima, desamparo e desesperança podem acompanhar essas pacientes se a dor persiste cronicamente ${ }^{5}$.

Apesar de ser muito freqüente, o fato de não se conhecer bem a fisiopatologia das mastalgias, na qual interagem fatores hormonais, alimentares, metabólicos e emocionais ${ }^{6}$, tem gerado indefinição quanto ao tipo de conduta assistencial preferencial a ser oferecido a essas mulheres. Existe grande número de opções terapêuticas ${ }^{7}$, das quais o próprio placebo tem uma resposta de $19 \%$ nos ensaios clínicos ${ }^{8}$. Obviamente, os aspectos éticos de se prescrever um placebo fora de ensaios clinicos devem ser questionados ${ }^{8}$.

Ao longo dos anos, têm sido usados os mais diferentes recursos e drogas com a finalidade de tratar a dor mamária: ácidos graxos essenciais ${ }^{3}$, dieta com restrições de metilxantinas ${ }^{9}$, vitami- 
nas $^{6}$, tamoxifeno $^{4}$, danazo $^{10}$, gestrinona ${ }^{11}$ e excepcionalmente os análogos do $\mathrm{LHRH}^{12}$.

Há diversos aspectos implicados no uso dessas drogas, como: custo elevado, eficácia nem sempre comprovada, efeitos colaterais às vezes significativos, além da recorrência da dor em metade das pacientes tratadas num período curto de tempo, após terem experimentado melhora e suspendido o tratamento ${ }^{13}$. Por este motivo, resolvemos avaliar a eficiência do tratamento não-medicamentoso (orientação verbal) especificamente nas mastalgias cíclicas e a influência que o tempo de existência da sintomatologia pudesse de alguma forma exercer nesses resultados. A conduta proposta é simples, não acarreta maiores gastos e é totalmente desprovida de efeitos colaterais inerentes a algumas drogas.

\section{Pacientes e Métodos}

Foi realizado um trabalho prospectivo do tipo experimental não-controlado, com 128 mulheres com queixa inequívoca de mastalgia cíclica que procuraram ou foram encaminhadas ao ambulatório de Patologia Mamária, do Centro Integrado de Saúde Amaury de Medeiros (CISAM) da Faculdade de Ciências Médicas/UPE. Estas foram atendidas no período de 23 de dezembro de 1998 a 26 de agosto de 1999. Foram excluídas do estudo pacientes com patologia mamária detectável ao exame clínico ou após propedêutica pertinente (mamografia e/ou ultra-sonografia) ou que estivessem fazendo uso de drogas que pudessem de alguma maneira influir na dor mamária: anticoncepcional hormonal, tamoxifeno, bromocriptina, etc. Foram também excluídas pacientes com dor referida na mama mas de causa extramamária e as portadoras de mastalgia acíclica.

A mastalgia cíclica (variável dependente) foi classificada em quatro diferentes categorias, levando-se em consideração a intensidade maior ou menor da dor: grau I (leve), grau II (moderado), grau III (intenso) e grau 0 (ausência de dor).

$\mathrm{Na}$ mastalgia grau I (leve), as mamas eram pouco dolorosas, a dor não interferia nas atividades habituais e não requeria analgésicos. Na mastalgia grau II (moderado), as mamas eram moderadamente dolorosas, a dor chegava a interferir com as atividades habituais e raramente requeria analgésicos. Na mastalgia grau III (intenso), as mamas eram intensamente dolorosas, a dor interferia nas atividades habituais e quase sempre necessitava de analgésicos. No grau 0 de mastalgia (ausência de dor), eram classificadas as pacientes que após tratamento não-medicamentoso (orientação verbal) sentiam-se curadas.

A orientação verbal fundamenta-se na abor- dagem sistemática dos seguintes aspectos: 1) explicação sobre a natureza não-neoplásica do sintoma; 2) a não-elevação do risco relativo para o câncer nas portadoras de dor mamária e 3) as possiveis causas do sintoma e a sua evolução natural. Tudo isso, após exame físico detalhado com propedêutica pertinente. Nesse tipo de abordagem, a relação médico-paciente é fundamental.

Para permitir um referencial comparativo pré e pós-tratamento, as pacientes submeteramse a uma avaliação basal da dor, que consistiu na verificação de parâmetros, de base subjetiva, informados pela paciente com supervisão e orientação do pesquisador:

$1^{\circ}$ - Classificação sintomática da dor mamária em graus I, II ou III (anamnese). Parâmetro básico, alicerce do estudo; $2^{\circ}$ - Atribuição de nota para a intensidade da dor mamária pela própria paciente de 0 a 10 (nota 10 para a dor máxima) parâmetro secundário, usado como apoio; $3^{\circ}$ - Avaliação da intensidade da dor mamária, mediante uso da escala linear analógica visual da dor, que foi apresentada à paciente pelo pesquisador parâmetro secundário, também usado como apoio.

Para a análise evolutiva do quadro doloroso foi utilizado o CBS - "Cardiff Breast Score" modificado $^{14}$, que consistia na autoavaliação da paciente em relação à sua sintomatologia dolorosa. Após a autoavaliação a evolução foi categorizada em: cura - quando não há qualquer dor residual após tratamento; melhora - quando permanece alguma dor residual, mas considerada pela paciente perfeitamente tolerável; nenhuma resposta - a dor permanece inalterada e considera-se piora quando há um agravamento dos índices da sintomatologia.

A eficácia do tratamento não-medicamentoso foi constatada quando pelo menos três dos quatro parâmetros (classificação sintomática da dor, nota, escala linear analógica visual da dor, análise evolutiva do quadro doloroso) utilizados com essa finalidade mostrassem diminuição de intensidade da dor em qualquer nivel.

A nota, a escala analógica visual da dor e a classificação em graus I, II e III em relação à intensidade da dor mamária, depois de estabelecidas, permaneceram inacessiveis ao entrevistador na consulta de reavaliação do tratamento usado até a análise final dos resultados.

O presente trabalho foi submetido à Comissão de Ética em Pesquisa do CISAM e aprovado.

\section{Resultados}

Das 227 pacientes selecionadas para participar do estudo, satisfazendo, portanto os critérios de inclusão e exclusão, 128 retornaram para reavaliação final efetiva, constituindo a casuística 
de fato. A distribuição dessas pacientes em relação ao grau de intensidade da dor mamária antes do tratamento não-medicamentoso (orientação verbal) está exposta na Tabela 1. Observa-se que a maioria das pacientes apresentava mastalgia moderada.

Tabela 1 - Distribuição das pacientes com mastalgia cíclica de acordo com o grau de intensidade da dor antes do tratamento não-medicamentoso (orientação verbal).

\begin{tabular}{lcc}
\hline Graus de intensidade da dor & \multicolumn{2}{c}{ Antes do tratamento } \\
& $\mathbf{n}$ & $\%$ \\
\hline Grau I (leve) & 45 & 35,1 \\
Grau II (moderado) & 66 & 51,7 \\
Grau III (intenso) & 17 & 13,2 \\
Total & 128 & 100,0 \\
\hline
\end{tabular}

A Tabela 2 mostra que um número maior $(61,7 \%)$ de pacientes apresentava mastalgia por um longo periodo de tempo, isto é, superior a 12 meses, independente do grau de mastalgia (grau I $=51,1 \%$; grau II $=68,1 \%$; grau III $=64,7$ ); no entanto, não houve diferença estatisticamente significante quando se comparou o grau I de mastalgia com os graus II e III, levando em consideração o tempo de existência da sintomatologia por mais de 12 meses ou por menos.

Numa visão inicial observamos que, após o tratamento não-medicamentoso (orientação ver- bal), 19 das pacientes $(14,8 \%)$ dos diversos grupos de intensidade da dor encontravam-se sem mastalgia na consulta de reavaliação (período médio de retorno $=$ dois meses e dez dias), conforme se observa na Tabela 3. O aumento no número de pacientes na mastalgia leve, de 45 para 74 , significa que pacientes dos dois grupos subseqüentes melhoraram com o tratamento proposto.

Foram consideradas curadas ou com melhora, segundo autoavaliação das pacientes (análise evolutiva do quadro doloroso), 66,7\% dos casos com grau I, $65,2 \%$ e $47 \%$ com graus II e III, respectivamente, como pode ser visto na Tabela 4. Não houve, porém, diferença estatisticamente significante quando comparamos as curadas e melhoradas de grau III de mastalgia com aquelas curadas e melhoradas de graus I e II.

Na Tabela 5, observa-se que metade das pacientes (64 de 128) evoluiu para mastalgia de menor intensidade após tratamento. Observa-se também que, no grupo originalmente constituído por pacientes com mastalgia grau III (intensa), aproximadamente dois terços $(64,8 \%)$ sofreram alteração da sintomatologia após o tratamento, havendo diminuição da intensidade da dor. A mudança para graus mais baixos de mastalgia nas pacientes que inicialmente se queixavam de dor de moderada intensidade (grau II) ocorreu em $69,8 \%$ dos casos, com diferença estatisticamente significante em relação aos outros grupos.

Tabela 2 - Distribuição das pacientes com mastalgia cíclica de diferentes graus de intensidade da dor, de acordo com o tempo de existência da sintomatologia.

\begin{tabular}{|c|c|c|c|c|c|c|c|c|}
\hline \multirow[t]{3}{*}{ Intensidade da dor } & \multicolumn{8}{|c|}{ Tempo de existência da mastalgia } \\
\hline & \multicolumn{2}{|c|}{$\leq 3$ meses } & \multicolumn{2}{|c|}{ 4-6 meses } & \multicolumn{2}{|c|}{ 7-12 meses } & \multicolumn{2}{|c|}{$>12$ meses } \\
\hline & $\mathrm{n}$ & $\%$ & $\mathrm{n}$ & $\%$ & $\mathrm{n}$ & $\%$ & $\mathrm{n}$ & $\%$ \\
\hline Grau I (leve) & 7 & 50,0 & 8 & 44,5 & 7 & 41,2 & 23 & 29,1 \\
\hline Grau II (moderado) & 5 & 35,7 & 9 & 50,0 & 7 & 41,2 & 45 & 56,9 \\
\hline Grau III (intenso) & 2 & 14,3 & 1 & 5,5 & 3 & 17,6 & 11 & 14,0 \\
\hline Total & 14 & 100,0 & 18 & 100,0 & 17 & 100,0 & 79 & 100,0 \\
\hline
\end{tabular}

$\chi^{2}=3,28 ; p=0,07$

Tabela 3 - Distribuição das pacientes com mastalgia cíclica de acordo com o grau de intensidade da dor, antes e após tratamento não medicamentoso (orientação verbal).

\begin{tabular}{lcccc}
\hline Intensidade da dor & \multicolumn{3}{c}{ Tratamento } \\
& \multicolumn{2}{c}{ Antes } & \multicolumn{2}{c}{ Depois } \\
& $\mathbf{n}$ & $\%$ & $\mathbf{n}$ & $\%$ \\
\hline Grau 0 (sem mastalgia) & - & - & 19 & 14,8 \\
Grau I (leve) & 45 & 35,1 & 74 & 57,9 \\
Grau II (moderado) & 66 & 51,7 & 26 & 20,3 \\
Grau III (intenso) & 17 & 13,2 & 9 & 7,0 \\
Total & 128 & 100,0 & 128 & 100,0 \\
\hline
\end{tabular}

Tabela 4 - Distribuição dos resultados da terapêutica não medicamentosa (orientação verbal) nos diferentes graus de mastalgia cíclica, segundo autoavaliação das pacientes.

\begin{tabular}{lcccccc}
\hline Auto-avaliação & \multicolumn{2}{c}{ Grau I } & \multicolumn{2}{c}{ Grau II } & \multicolumn{2}{c}{ Grau III } \\
das pacientes & $\mathbf{n}$ & $\%$ & $\mathbf{n}$ & $\%$ & $\mathbf{n}$ & $\%$ \\
\hline Curou & 7 & 15,5 & 12 & 18,1 & - & - \\
Melhorou & 23 & 51,2 & 31 & 47,1 & 8 & 47,0 \\
Inalterado & 15 & 33,3 & 21 & 31,8 & 9 & 53,0 \\
Piorou & - & - & 2 & 3,0 & - & - \\
Total & 45 & 100,0 & 66 & 100,0 & 17 & 100,0 \\
\hline$\chi^{2}=2,20 ; p=0,13$ & & & & & &
\end{tabular}


Tabela 5 - Distribuição das pacientes com mastalgia cíclica, de acordo com a mudança de graus de intensidade da dor mamária, após tratamento não-medicamentoso (orientação verbal)

\begin{tabular}{|c|c|c|c|c|c|c|}
\hline \multirow[t]{3}{*}{ Após tratamento } & \multicolumn{6}{|c|}{ Graus de intensidade da dor } \\
\hline & \multicolumn{2}{|c|}{$\begin{array}{c}\text { Grau I } \\
(n=45)\end{array}$} & \multicolumn{2}{|c|}{$\begin{array}{l}\text { Grau II } \\
(n=66)\end{array}$} & \multicolumn{2}{|c|}{$\begin{array}{l}\text { Grau III } \\
(n=17)\end{array}$} \\
\hline & $\mathrm{n}$ & $\%$ & $\mathrm{n}$ & $\%$ & $\mathrm{n}$ & $\%$ \\
\hline Grau 0 & 7 & 15,5 & 12 & 18,1 & - & - \\
\hline Grau I & 32 & 71,2 & 34 & 51,7 & 8 & 47,2 \\
\hline Grau II & 6 & 13,3 & 17 & 25,7 & 3 & 17,6 \\
\hline Grau III & - & - & 3 & 4,5 & 6 & 35,2 \\
\hline Total & 45 & 100,0 & 66 & 100,0 & 17 & 100,0 \\
\hline
\end{tabular}

$\chi^{2}=20,98 ; p=0,001, \operatorname{RR}=2,30($ IC $95 \%: 1,55-3,41)$

O tempo de existência da sintomatologia alterou o resultado do tratamento não-medicamentoso das mastalgias cíclicas nos diversos graus de intensidade da dor. As pacientes que apresentavam a sintomatologia por um período de tempo mais longo (>12 meses) responderam menos satisfatoriamente ao tratamento, mas não se observou uma diferença estatisticamente significante entre esse grupo e os demais.

Em resumo, em $76(59,4 \%)$ das 128 pacientes estudadas, comprovou-se a eficiência do tratamento. Foi sugerida uma eficiência diretamente proporcional à maior intensidade da sintomatologia, porém não houve diferença estatisticamente significante quando comparamos pacientes com graus II e III de mastalgia com as de grau I.

\section{Discussão}

Nosso estudo avaliou os resultados da conduta não-farmacológica (orientação verbal), especificamente em pacientes com mastalgia cíclica, por se tratar da forma mais freqüente de mastalgia e ser a que responde melhor aos tratamentos convencionais propostos ${ }^{14}$.

O índice de eficiência obtido por nós foi de $59.4 \%$ de uma forma global, porém variando nos casos de mastalgia leve (grau I; 51,1\%), moderada (grau II; 63,6\%) e intensa (grau III; 64,7\%), mas não houve diferença estatisticamente significante entre esses grupos. Pesquisadores ingleses ${ }^{15}$ referiram $85 \%$ de sucesso no alivio da dor mamária com a orientação verbal (reassurance).

Uma orientação adequada indicando que a condição é benigna permite pelo menos que $85 \%$ das pacientes aceitem e tolerem a sua dor mamária sem medicação ${ }^{14}$. Consideramos esse aspecto fundamental, pois põe em evidência que o sucesso do tratamento não significa necessariamente a cura total da dor, mas a sua melhora a níveis toleráveis. Gateley et al. ${ }^{14}$ consideram uma resposta clínica útil mesmo que persista alguma dor residual, porém considerada pela paciente facilmente tolerável. Barros et al. ${ }^{16}$ obtiveram sucesso com esse método em $70,2 \%$ dos casos e recomendam esta medida como conduta de eleição para mulheres com mastalgia.

A menor eficiência da orientação verbal nos nossos achados, em relação aos resultados obtidos pelos autores ingleses, talvez possa ser explicada pelo fato de que a população atendida no nosso ambulatório é predominantemente constituida por mulheres com formas moderadas ou intensas de mastalgia (64,9\%), para as quais os resultados não são tão satisfatórios ${ }^{16}$. A mastalgia severa, por exemplo, tem um curso crônico de recidivas, freqüentemente requerendo repetidos tratamentos com drogas ${ }^{17}$.

$\mathrm{Na}$ nossa casuística as pacientes com mastalgia intensa e moderada se beneficiaram mais com o tratamento do que as portadoras das formas leves de mastalgia, porém não se observou diferença estatisticamente significante entre esses grupos. Pode-se ainda especular que as pacientes que não voltaram (227 foram selecionadas e somente o número suficiente para a amostra de 128 retornou, boa parte delas convocadas por cartas) para reavaliação obtiveram melhora e, por isso mesmo, não voltaram ao ambulatório. Este comportamento já havia sido sugerido por McFayden et $a .^{18}$, em trabalho realizado em Edinburgh. Outra explicação é que as pacientes simplesmente não se adaptaram ao tratamento proposto, pelo fato de nossa cultura ser fortemente voltada para o tratamento farmacológico nas mais variadas circunstâncias, impossibilitando assim a avaliação do tratamento usado.

Pode-se considerar ainda a possibilidade levantada por McFayden et al. ${ }^{18}$ de que o tratamento não-medicamentoso (orientação verbal) pode não alcançar todo o sucesso esperado na primeira consulta, pois requer tempo considerável com a repetição de explicações da causa provável da dor e grande paciência. É pertinente colocar em evidência que, nos nossos índices de eficiência com a terapêutica não-medicamentosa, estão incluídas pacientes que aparentemente ficaram curadas como também outras que sentiram melhora, apesar de permanecerem com alguma dor residual, equivalente a CBS II, escore usado na "Cardiff Mastalgia Clinic" e que significa melhora com dor residual facilmente tolerável pela paciente ${ }^{14}$. Ainda é prudente lembrar que em algumas pacientes os sintomas mamários irão desaparecer independentemente de qualquer terapêutica, como foi muito bem observado por Fentiman e Hamed ${ }^{4}$, que afirmaram: "Para a maioria das pacientes com mastalgia cíclica a condição dura por dois a três meses e cura espontaneamente. Nessas circunstâncias, nenhum 
tratamento é necessário". Considerar ainda o fato de que a dor varia em severidade de ciclo a ciclo ${ }^{19}$.

No nosso estudo, 25\% das pacientes apresentavam sintomatologia dolorosa das mamas por um período de tempo considerado curto (seis meses ou menos). Poderiam, portanto, ser excluidas de participação se os critérios de elegibilidade assim o determinassem. Fentiman et al. ${ }^{20}$ e Sismondi et al. ${ }^{21}$, por exemplo, recomendam que a terapêutica medicamentosa deve ser reservada para aqueles casos nos quais os sintomas duram mais que seis meses. Por outro lado, a ausência absoluta de efeitos colaterais, contra-indicações e custo financeiro da orientação verbal viabilizaram a inclusão dessas pacientes sem a preocupação com aspectos éticos que poderiam eventualmente estar envolvidos e que são tão importantes, particularmente nos estudos experimentais. Outro aspecto a considerar é que grande parte das nossas pacientes $(61,7 \%)$ eram portadoras de mastalgia cíclica por um longo período de tempo (>12 meses) e eram jovens (a faixa etária entre 20 e 29 anos representava o grupo maior), significando que a expectativa de cura ou melhora não era facilmente esperada, segundo dados da literatura. O início de dor cíclica na mama, antes da idade de 20 anos, é seguido normalmente por um longo período de tempo sintomático. A idade do início da sintomatologia permite predizer, de alguma maneira, o curso da doença. Pacientes jovens com sintomas severos devem esperar muitos anos de sintomatologia ${ }^{17}$.

Na nossa casuística, foram incluidas mulheres classificadas como portadoras da forma leve $(35,1 \%)$, forma moderada $(51,7 \%)$ e forma intensa $(13,2 \%)$ de mastalgia, contrastando também com a prevalência do sintoma em uma população de universitárias do Estado de São Paulo, que foram classificadas da seguinte forma: $91,8 \%$ de forma leve, 6,8\% de forma moderada e 1,2\% de forma intensa ${ }^{22}$. Por outro lado, quando questionadas diretamente, $45 \%$ das mulheres referiram dor suave nas mamas e $21 \%$ dor severa ${ }^{15}$.

O predominio da forma moderada (grau II) de mastalgia em nossa casuística em relação à de outros autores pode ser explicado pelo fato de, neste estudo, ter-se pesquisado detalhadamente a possibilidade de interferência da dor mamária nas atividades normais das pacientes. Na classificação das mastalgias usada na pesquisa, esse era um aspecto fundamental, levando algumas pacientes ora para a forma moderada, ora para a forma severa de mastalgia. Essas diferenças também poderiam ser explicadas pelo fato de que nossa amostragem foi constituída de pacientes referidas ao nosso ambulatório especializado.

Visando tornar mais objetivos e de certa forma quantitativos os efeitos sobre um sintoma altamente subjetivo como é a dor, usamos sistematicamente a escala linear analógica visual da dor
(EAVD) antes e após o tratamento, escala esta considerada como o melhor método para avaliar a severidade da dor objetivamente ${ }^{4}$.

$\mathrm{Na}$ classificação sintomática da dor em graus, o fato de a dor interferir ou não nas atividades normais como sono, trabalho e vida sexual, promove também uma útil análise da severidade da dor ${ }^{23}$. Essa classificação também foi usada sistematicamente, antes e após o tratamento não-farmacológico. A nota atribuida pela paciente, antes e após o tratamento, e usada também para avaliar a severidade da dor e a resposta ao tratamento, é eqüivalente aos resultados obtidos com a EAVD, podendo ser dispensada em novas avaliações. Observamos que, em 89\% das vezes, a nota atribuída à intensidade da dor corresponde ao mesmo número da escala analógica visual da dor apontada pela paciente.

A explicação sobre como a orientação verbal permite obter tão bons resultados no alivio da dor mamária passa seguramente por aspectos psicológicos. Sabe-se que sintomas somáticos que levam pacientes a departamentos médicos e cirúrgicos podem ser manifestação de estados de ansiedade e de depressão e que seguramente não têm base em patologia orgânica ${ }^{24}$.

Finalmente, o tempo decorrido entre a primeira consulta (com o efeito inicial do tratamento não-medicamentoso) e a reavaliação do tratamento subseqüente pode, de alguma maneira, ter influenciado os resultados, obrigando a um estudo mais aprofundado sobre esse aspecto posteriormente. A tentativa de diminuir vieses de informação usando vários critérios para avaliar a intensidade da dor antes e após o tratamento não-medicamentoso pareceu extremamente válida porque informações contraditórias, ora observadas na pontuação da EAVD, ora na atribuição da nota pelas pacientes, além da dificuldade às vezes encontrada para classificar a dor em diferentes graus de intensidade, foram mais freqüentes do que o esperado.

Quanto às pacientes que precisaram de propedêutica complementar como mamografia e/ ou ultra-sonografia, observou-se que foram submetidas a esses exames somente 9,3\% das pa-cientes, a maioria com mastalgia de leve intensidade, grau I $(66,6 \%)$. Sentiram-se curadas ou melhoraram na análise evolutiva do quadro doloroso (autoavaliação) 58,3\%, também melhorando nos demais parâmetros (diminuindo a nota e alterando a escala analógica visual para menos). O valor que tais exames possam ter exercido em relação a proporcionarem ou não uma maior eficiência ao tratamento é dificil de avaliar, considerando que essas pacientes eram tratadas, na primeira consulta, com a orientação verbal e só depois voltavam para reavaliação com os resultados. Na realidade, se eles tivessem de influir o fariam provavelmente negativamente, considerando a expectativa gerada antes de sua realização até o resultado final. 
Concluímos que a conduta não-medicamentosa (orientação verbal) deve ser tentada sempre como a primeira opção terapêutica para mulheres com mastalgia cíclica (indice de sucesso de $59,4 \%)$. Não houve diferença estatisticamente significante em termos de eficiência do método entre os grupos que apresentavam mastalgia leve, moderada ou intensa. O tempo de existência da sintomatologia dolorosa não alterou os resultados.

\section{SUMMARY}

Purpose: to evaluate the nonpharmacologic treatment (reassurance) as a first line therapy for women with cyclical mastalgia, and to observe if a prolonged course of pain alters the outcome.

Methods: we conducted a noncontrolled experimental study with a sample of 128 eligible women with a clear history of cyclical mastalgia treated with reassurance. A visual linear analogical scale of the pain was used before and after treatment in order to assess its severity and the mastalgias were classified into degrees I (mild), II (moderate) and III (severe) according to the intensity of pain. We also used a modified Cardiff Breast Score (CBS) to assess the clinical response. The data analysis was performed using the $\chi^{2}$ test (Epi-Info 6.04 software).

Results: we verified a success rate of $59.4 \%$ with reassurance, but there was no significant statistical difference between the groups $(p=0.16)$ with different degrees of mastalgia. The less satisfactory response to the nonpharmacologic treatment in those pacients with a prolonged course of pain was only apparent, since there was no significant statistical difference $(p=0.14)$.

Conclusion: reassurance should be always tried as the first choice treatment for women with cyclical mastalgia, independently of pain intensity. Prolonged course of pain did not alter the outcome.

KEY WORDS: Breast: benign diseases. Mastalgia, reassurance.

\section{Referências}

1. Gebrim LH, Lima GR, Nazário ACP, Kemp C. New concepts in breast pain. In: Figueira ASS, Dias EN, Silva HMS, Barbosa CSD, editors. Mastology: breast disease. 1st ed. Amsterdam: Elsevier; 1995. p.12-5.

2. Souza GA. Mastalgia. Femina 1995; 23:311-6.

3. Steinbrunn BS, Zera RT, Rodriguez JL. Mastalgia. Tailoring treatment to type of breast pain. Postgrad Med 1997; 102:183-98.

4. Fentiman IS, Hamed H. Benign breast disease: who and how to treat for mastalgia. In: Figueira ASS, Dias EN, Silva HMS, Barbosa CSD, editors. Mastology: breast disease. $1^{\text {st }}$ ed. Amsterdam:
Elsevier; 1995. p.10-1.

5. Fox H, Walker LG, Heys SD, Ah-See AK, Eremin O. Are patients with mastalgia anxious, and does relaxation therapy help? Breast 1997; 6:138-42.

6. Bellieu RM. Mastodynia. Obstet Gynecol Clin North Am 1994; 21:461-77.

7. Holland PA, Gateley CA. Drug therapy of mastalgia. What are the options? Drugs 1994; 48:709-16.

8. Gateley CA. The powerful placebo. Lancet 1991; 337:927.

9. Minton JP, Abou-Issa H, Reiches N, Roseman JM. Clinical and biochemical studies on methylxanthine-related fibrocystic breast disease. Surgery 1981; 90:299-304.

10.O'Brien PM, Abukhalil IE. Randomized controlled trial of the management of premenstrual syndrome and premenstrual mastalgia using luteal phase-only danazol. Am J Obstet Gynecol 1999; 180:18-23.

11.Peters F. Multicentre study of gestrinone in cyclical breast pain. Lancet 1992; 339:205-8.

12.Hamed H, Calleffi M, Chaudary MA, Fentiman IS. LHRH analogue for treatment of recurrent and refractory mastalgia. Ann R Coll Surg Engl 1990; $72: 221-4$.

13.Wetzig NR. Mastalgia: a 3 year Australian study. Aust N Z J Surg 1994; 64:329-31.

14.Gateley CA, Miers M, Mansel RE, Hughes LE. Drug treatments for mastalgia: 17 years experience in the Cardiff Mastalgia Clinic. J R Soc Med 1992; 85:12-5.

15.Maddox PR, Mansel RE. Management of breast pain and nodularity. World J Surg 1989; 13:699-705.

16.Barros ACSD, Mottola Junior J, Ruiz CA, Borges MN, Pinotti JA. Resultados da conduta não medicamentosa (orientação verbal) para tratamento da mastalgia. Rev Bras Mastol 1997; 7:3-8.

17.Davies EL, Gateley CA, Miers M, Mansel RE. The long-term course of mastalgia. J R Soc Med 1998; 91:462-4.

18.McFayden IJ, Forrest AP, Chetty U, Raab G. Cyclical breast pain - some observations and the difficulties in treatment. Br J Clin Pract 1992; 46:161-4.

19.Mansel RE. ABC of breast diseases. Breast pain. BMJ 1994; 309:866-8.

20.Fentiman IS, Caleffi M, Brame K, Chaudary MA, Hayward JL. Double-blind controlled trial of tamoxifen therapy for mastalgia. Lancet 1986; 1:287-8.

21.Sismondi P, Giai M, Biglia N, Roagna R, Ponzoner R, Sgro L. Medical treatment of benign breast disease. In: Figueira ASS, Dias EN, Silva HMS, Barbosa CSD, editors. Mastology: breast disease. $1^{\text {st }}$ ed. Amsterdam: Elsevier; 1995. p.16-22.

22.Barros ACSD, Adamek EV, Muller AP, et al. Perfil epidemiológico da mastalgia em uma população universitária brasileira. Rev Bras Mastologia 1995; 5:24-30.

23. Gateley CA, Mansel RE. Management of cyclical breast pain. Br J Hosp Med 1990; 43:330-2.

24.Downey HM, Deadman JM, Leinster SJ. Psychological characteristics of women with cyclical mastalgia. Br J Surg 1990; 77:A705. 